\title{
La Dobroudja au carrefour des Europes
}

Dobruja at the Crossroad of Many Europes

Dobrogea la răscrucea mai multor Europe

\section{Bernard Lory}

\section{OpenEdition \\ Journals}

Édition électronique

URL : https://journals.openedition.org/ceb/9573

DOI : 10.4000/ceb.9573

ISSN : $2261-4184$

Éditeur

INALCO

Édition imprimée

ISBN : 978-2-85831-239-9

ISSN : 0290-7402

Référence électronique

Bernard Lory, "La Dobroudja au carrefour des Europes », Cahiers balkaniques [En ligne], 44 | 2016, mis en ligne le 27 novembre 2017, consulté le 07 juillet 2021. URL : http://journals.openedition.org/ceb/ 9573 ; DOI : https://doi.org/10.4000/ceb.9573

Ce document a été généré automatiquement le 7 juillet 2021.

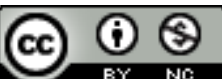

Cahiers balkaniques est mis à disposition selon les termes de la Licence Creative Commons Attribution - Pas d'Utilisation Commerciale 4.0 International. 


\title{
La Dobroudja au carrefour des Europes
}

\author{
Dobruja at the Crossroad of Many Europes \\ Dobrogea la răscrucea mai multor Europe
}

\section{Bernard Lory}

1 La Dobroudja est une région facile à définir sur la carte géographique, délimitée qu'elle est par la mer Noire à l'Est et par le cours du Danube au Nord et à l'Ouest; seule sa limite méridionale est sujette à discussion; il est en général admis qu'elle ne saurait dépasser la ligne Ruse-Varna. De nos jours, cette région naturelle est partagée entre deux États : la Roumanie possède la Dobroudja du Nord (Dobrogea en roumain), dont Constanţa est la capitale économique et la Bulgarie, la Dobroudja du Sud (Dobrudža en bulgare), dont Dobrich (Tolbuhin à l'époque communiste, Hacioğlu Pazarcik à l'époque ottomane) est le chef-lieu.

2 Dans cette esquisse, nous nous efforcerons de cerner les diverses facettes de cette région selon trois approches successives. Dans une vision géographique, nous nous demanderons d'abord comment on atteint la Dobroudja, au bout de quel itinéraire la situer? Puis, chaussant les lunettes de l'historien, nous tenterons d'établir le type de formations politiques qui ont pu contrôler ou exercer leur influence sur cette région. Notre troisième partie relève de l'anthropologie ou de la microhistoire; elle se penchera sur la destinée d'une famille de Tulcea, dont les positionnements sociaux entre la guerre de Crimée et la Première Guerre mondiale connaissent des ajustements intéressants à suivre, entre une ascendance bulgare, une volonté d'hellénisation et la nécessaire négociation avec le pouvoir roumain. 


\section{Un coup d'œil de géographe}

\section{Une Dobroudja danubienne}

3 Pour le regard occidental, il n'y a guère d'hésitation : la Dobroudja se situe à l'extrémité du cours du Danube. La grande voie d'eau qui parcourt l'Europe de Regensburg à Sulina sur $2380 \mathrm{~km}$ navigables pour les péniches de gabarit international (des chalands de faible tonnage partaient autrefois d'Ulm) dessert successivement Vienne, Bratislava, Budapest et Belgrade. La mer Noire constitue l'aboutissement ultime de cette navigation. Mais le petit massif des monts de Măcin oppose un obstacle et oblige l'énorme fleuve à contourner la Dobroudja, par un détour de quelque $400 \mathrm{~km}$. La question du percement d'un canal à travers la Dobroudja, entre Cernavodă et ConstanŢa, fut évoquée dès les années 1830 et tout particulièrement au moment de la guerre de Crimée. Le projet fut entrepris après la Seconde Guerre mondiale par la République populaire de Roumanie, qui y affecta une main-d'œuvre de prisonniers politiques qui en conservèrent un souvenir abominable. Il rencontra des difficultés techniques imprévues et dut être abandonné. Mais il ne s'agissait pas d'un projet absurde, voire criminel, comme le présente parfois un discours tendancieux, qui dénie toute rationalité aux régimes communistes. Repris sur d'autres bases techniques (en particulier un profil très évasé), le canal fut inauguré en 1984. Il raccourcit sensiblement le trajet à la mer et favorise le développement du port de ConstanŢa-Sud, mais tend à marginaliser ceux de Tulcea et de Sulina.

La voie danubienne fut privilégiée sous l'Empire romain, car elle permettait le transport rapide de troupes le long du limes, cette ligne défensive face aux Barbares d'Europe du Nord. Charlemagne en reconnut l'importance et entreprit de creuser une liaison entre le Main et le Danube (fosses Caroline), projet colossal qui n'aboutit... qu'en 1992, avec l'inauguration de la liaison Rhin-Main-Danube, qui permet au transport fluvial moderne de traverser l'Europe de Rotterdam à Constanţa.

Cette lecture fluviale a ses limites : elle est très fortement orientée de l'amont vers l'aval, dans le sens du courant, et considère la Dobroudja comme un terme, un lieu d'aboutissement, un déversoir pour la production de l'Europe centrale. La remontée du Danube n'était pas impossible pour la navigation à voile, en particulier dans les secteurs à faible pente comme celui de Dobroudja, de Valachie ou de Hongrie, mais elle était lente et très irrégulière. L'arrivée de la navigation à vapeur en 1834 constitua une petite révolution: dorénavant, on put circuler aussi bien vers l'amont que vers l'aval avec beaucoup de régularité. Les navires de la Donaudampfschiffahrtsgesellschaft (longtemps le mot le plus long de la langue allemande !) de Vienne scandaient par leurs passages hebdomadaires la vie des petits ports danubiens comme Cernavodă ou Silistra. L'Empire des Habsbourg, tout comme le Troisième Reich, fit du Danube l'axe majeur de leur pénétration vers le Sud-Est.

\section{Une Dobroudja pontique}

On peut considérer la Dobroudja comme une portion du littoral de la mer Noire et cette lecture inclut même son côté septentrional, puisque le cours final du Danube est accessible aux navires de mer, jusqu'à GalaŢi, port moldave, et Brăila, port valaque, en utilisant l'anaphore, contre-courant produit par le déplacement des énormes masses 
d'eau du fleuve. Cette lecture met la Dobroudja dans une perspective méditerranéenne, dominée par la navigation maritime. On peut remonter au vi siècle av. J.-C. avec les fondations de colonies grecques comme Odessos (Varna), Dionysopolis (Balčik), Bizonè (Kavarna), Callatis (Mangalia), Tomis (Constanţa) et Istros. Elles sont toutes de fondation ionienne, à l'exception de Callatis, colonie d'Héraclée du Pont, elle-même colonie dorienne. Ce dernier point indique que la Dobroudja peut aussi entrer dans une logique strictement pontique, liée au seul bassin de la mer Noire, même si, dans l'ensemble, c'est le tropisme méditerranéen qui prédomine.

7 Un rôle-clé se trouve dévolu à la puissance contrôlant le passage des Détroits : à partir de 330, la ville de Constantinople devient le centre de référence pour la Dobroudja et le restera durant tout le millénaire byzantin, puis, sous le nom d'Istanbul, pendant cinq siècles supplémentaires. Les thalassocraties italiennes, Venise et surtout Gênes, auront pendant plusieurs siècles des comptoirs éloignés en mer Noire. Après 1453 , ce commerce international cesse et la mer Noire se transforme en «lac turc»; les Occidentaux n'y reprendront pied qu'à la fin du XVIII ${ }^{e}$ siècle.

Cette approche maritime de la Dobroudja est très largement conditionnée par un facteur physique : le passage des Détroits. Or, le passage du Bosphore n'est pas aisé dans le contexte de la navigation à voile, car il est parcouru par un fort courant de surface, orienté nord-sud. Il sera donc toujours plus facile d'aller du bassin pontique vers le bassin méditerranéen que l'inverse. Cela détermine les échanges interrégionaux: le bassin pontique sera exportateur de matières premières (principalement des céréales, mais aussi du poisson séché, des fourrures, des esclaves, du bois de construction) ; en échange, il recevra des produits plus élaborés du bassin méditerranéen (vin, huile d'olive, céramique, orfèvrerie). Ce caractère de périphérie économique dévolu au bassin pontique s'observe dès l'Antiquité, et se maintient dans le cadre des Empires byzantin et ottoman. La Dobroudja, dénommée Petite Scythie dans l'Empire romain, fut un lieu d'exil et pendant deux mille ans les plaintes d'Ovide lui vaudront une triste réputation dans les milieux cultivés.

\section{Une Dobroudja steppique}

9 Ce nom de Petite Scythie rappelle opportunément qu'immédiatement au nord du Danube s'ouvrent les espaces immenses de la steppe eurasiatique et que l'on peut, à bride abattue, galoper jusqu'à la Grande Muraille sans rencontrer d'autres obstacles que des fleuves. C'est le monde des cavaliers et des grands troupeaux, des empires nomades qui surgissent on ne sait d'où et disparaissent brusquement. Point de villes d'ici à Cambaluc...

Mais la Dobroudja ne fait-elle pas partie de monde-là ? Les botanistes observent la jonction des espèces spécifiques à la steppe avec les représentants les plus septentrionaux de la flore méditerranéenne. Le pastoralisme nomade y fut longtemps prédominant et, jusqu'à la fin du XIX ${ }^{\mathrm{e}}$ siècle, des bergers tatars menaient leurs troupeaux sur le plateau herbeux entre Danube et mer Noire. Extrême limite de la steppe, la Dobroudja fut un lieu de sédentarisation pour des vagues successives de nomades. On pense tout particulièrement aux Protobulgares $\mathrm{du} \mathrm{VII}^{\mathrm{e}}$ siècle; les chercheurs se disputent autour de la localisation de l'Ongăl, leur premier établissement danubien. 
11 La Dobroudja a connu différents peuples de langues turciques, tels les Protobulgares et les Coumans, avant que n'arrivent les Turcs ottomans. Il semble qu'une colonisation de Turcs seldjoukides soit à l'origine des Gagaouzes, ces chrétiens turcophones du littoral pontique occidental. Au XIV ${ }^{e}$ siècle, une principauté de Dobroudja s'émancipe du Deuxième Royaume bulgare; son premier prince porte le nom turc de Balik, son successeur le nom slave de Dobrotica, duquel les Turcs ont dérivé le nom de Dobroudja. La présence de musulmans hétérodoxes (Kăzălbaši) est attestée au début du $\mathrm{Xv}^{\mathrm{e}}$ siècle. Durant toute la période ottomane, la région est principalement peuplée de Turcs et la présence chrétienne, slave, roumaine ou grecque $\mathrm{y}$ reste très discrète. Pour l'Empire ottoman, la Dobroudja est le maillon indispensable qui assure la liaison continentale avec l'État vassal musulman des Tatars de Crimée.

Cet ancrage géographique connaît pourtant ses limites : la progression de l'agriculture, la densification de la population, le développement d'un maillage urbain mettent peu à peu fin à un mode de vie plurimillénaire. Derrière la conurbation qui s'établit entre Constanţa et Cernavodă, il est difficile aujourd'hui d'imaginer les solitudes pastorales d'il y a cent cinquante ans.

\section{La Dobroudja et son hinterland carpato-valaque}

13 La vision que cherche à promouvoir l'État roumain est moins convaincante d'un point de vue strictement géographique. La Dobroudja du Nord constitue, géologiquement parlant, un isolat radicalement différent de son environnement, sans aucun rapport avec l'arc carpatique. Les monts de Măcin sont en effet un vestige du lointain plissement hercynien; il est paradoxal que ce fragment le plus ancien de la croûte terrestre soit au contact immédiat des sédiments les plus jeunes, les dépôts alluviaux du delta du Danube.

14 L'historiographie roumaine insiste sur un épisode du xIV siècle, le règne de Mircea l'Ancien, qui rattacha effectivement la Dobroudja à la Valachie, peu avant qu'elle ne devienne durablement ottomane. Force est pourtant de constater que l'histoire de la Dobroudja s'intègre mal au grand récit national, et que la région, une fois passée la période romaine (et l'incontournable Tropaeum Traiani d'Adamclisi), est laissée de côté. L'histoire de la Dobroudja, qui s'appuie sur les archives ottomanes, nécessite en effet des historiens de formation spécifique.

Le Danube dresse-t-il donc une barrière si infranchissable ? Le fleuve, en lui-même, n'a jamais constitué un obstacle et l'on a toujours beaucoup circulé entre ses deux rives. La frontière ethnique n'est qu'approximative et on retrouve une minorité roumaine au sud du fleuve, de même qu'une minorité bulgare au nord. Plus que le fleuve lui-même, d'ailleurs, il semble que ce soit la forêt marécageuse le bordant au nord (luncă, baltă) qui constituait l'obstacle le plus difficile à franchir. Les ports importants du Danube inférieur sont situés sur la rive méridionale surélevée, c'est-à-dire en Bulgarie. Les deux ports roumains, Brăila et GalaŢi, sont situés au nord du coude dobroudjan, et, comme nous l'avons dit, directement reliés à la mer. Somme toute, si l'on souhaite traverser le grand fleuve, on choisira plutôt un autre secteur que la Dobroudja. Sauf, bien sûr, si ce sont les pâturages d'entre mer Noire et Danube qui sont le but du voyage : au XIX siècle, les mocani, bergers transylvains, passaient chaque année avec leurs troupeaux en territoire ottoman. 
L'orientation change radicalement à partir de 1878. Il faut alors « coudre » la Dobroudja au Vieux-Royaume. Ce sera le pont de Cernavodă, fleuron de l'architecture civile du jeune État roumain, inauguré en 1895, et permettant la liaison ferroviaire Bucarest-ConstanŢa. À partir de là, la façade maritime reliée à son hinterland peut jouer tout son rôle économique. Cette synergie ne cesse se renforcer tout au long du $\mathrm{xx}^{\mathrm{e}}$ siècle. Elle constitue de nos jours une tranquille évidence.

\section{Un coup d'œil d'historien}

\section{La logique des comptoirs}

17 La première forme de contrôle politique attesté sur la région est la fondation des comptoirs grecs de l'époque classique. Ils se contentent d'installations ponctuelles, réparties environ tous les $40 \mathrm{~km}$, le long du littoral pontique, partout où un golfe, un cap protecteur viennent échancrer une côte assez peu accueillante. Ce sont de petites enclaves de civilisation méditerranéenne qui se sont transportées avec leurs dieux, leurs institutions, leur langue, leurs usages. Les liens établis avec la population locale sont de nature essentiellement commerciale. La structuration politique des Gètes et des Thraces est assez rudimentaire, tout au moins avant l'époque hellénistique. Ce schéma thalassocratique se retrouve au Moyen-Âge avec les comptoirs génois, implantés à l'embouchure des grands fleuves du bassin pontique (et tout particulièrement Chilia). Il est aussi caractéristique de l'Empire byzantin dans sa dernière phase. Il explique en grande partie le maintien d'une population grecque sur ce rivage. Il faut sans doute renoncer à une vision romantique qui exalte l'enracinement millénaire de ces colonies, mais plutôt y voir une diaspora constamment alimentée par des apports nouveaux de marins et de commerçants, compensant son assimilation progressive dans la population environnante majoritaire. Dans cette histoire, les regards sont tournés vers la mer.

\section{La logique des Empires}

La logique des Empires est continentale. Leur expansion à l'ensemble de l'œkoumène reste une vue de l'esprit : la gestion des espaces a ses contraintes propres et il faut se poser des limites raisonnables. Il est frappant de constater que le Danube inférieur marque la limite septentrionale des constructions impériales successives. Alexandre le Grand, dont l'esprit de démesure est resté légendaire, ne l'a pas dépassé. L'Empire romain a la Petite Scythie pour limite extrême. L'Empire byzantin, au cours de ses longues fluctuations territoriales, rétablit sa frontière danubienne sous Justinien, puis à nouveau sous les Comnène, sous le nom de province du Paristrion. L'Empire ottoman envoie de nombreuses armées ravager les territoires nord-danubiens, mais s'abstient de les incorporer, en préférant un système d'administration indirecte (à l'exception de quelques enclaves urbaines); la Dobroudja fait partie, entre 1864 et 1878, du vilayet du Danube, province où l'Empire ottoman procède, à titre expérimental, à des réformes modernisatrices hardies. Dans ces quatre cas, la construction impériale se fait à partir du sud, à partir d'un noyau méditerranéen. La Dobroudja se retrouve en situation d'appendice septentrional, vulnérable aux attaques venant des territoires voisins. 
19 Mais on peut aussi inverser la perspective et envisager la construction impériale à partir du nord. Le vallum dobroudjan, longtemps désigné comme «mur de Trajan », a été en réalité construit par une Dobroudja «barbare», afin de se protéger d'attaques venant de l'Empire byzantin. La «Rus » de Kiev s'étend vers le sud sous Svjatoslav et vient, en 969, dévaster Preslav, siège des rois bulgares (épisode qu'on évitait de mentionner à l'époque de "l'immarcescible amitié bulgaro-soviétique»). Si cette offensive russe est brève, le contrôle de la Horde d'or sur le Bas-Danube et le Deuxième Royaume bulgare, s'étend sur toute la seconde moitié du xIII siècle. L'Empire russe, enfin, progresse inexorablement vers les bouches du Danube à partir de la fin du XVIII ${ }^{e}$ siècle. Les guerres se succèdent tous les vingt ans environ : 1768-1774, 1789-1792, 1806-1812, 1828-1829, 1853-1856, 1877-1878. À l'exception de la dernière, toutes ces guerres se déroulent (au moins partiellement) en Dobroudja. La frontière russo-ottomane s'établit sur le Danube, au bras de Chilia, en 1812 ; de 1829 à 1856, elle descend même sur le bras Saint-Georges. L'Empire soviétique aura aussi sa frontière sud-ouest sur le bras de Chilia; le port danubien le plus actif, dans la deuxième moitié du $x^{e}$ siècle, est celui de Reni, de création récente.

20 La logique impériale encourage le brassage des populations. Pour densifier la population dans la zone de steppe, la Russie et l'Empire ottoman encouragent l'implantation de colons d'origines variées: Allemands, Bulgares, Juifs en Bessarabie ; Russes vieux-croyants, Allemands, Tatars criméens, Tcherkesses en Dobroudja. La mosaïque ethnoconfessionnelle qu'on observe sur les bords nord-ouest de la mer Noire à la fin du XIX ${ }^{e}$ siècle est le résultat de ces politiques, qui visent avant tout à densifier la population, et non pas à diluer l'élément roumain ou bulgare comme le prétendent les historiographies nationalistes.

\section{La logique des États-nations}

21 La Dobroudja est entraînée assez tardivement dans la logique des États-nations, au moment du traité de Berlin en 1878. Deux pays entrent alors en compétition pour sa possession : la Roumanie, dont l'indépendance vient d'être reconnue, et la Bulgarie, qui n'a encore que le statut de principauté vassale de l'Empire ottoman. Les diplomates doivent trancher et fixer une frontière. La proposition russe du traité de San Stefano accorde Mangalia à la principauté bulgare; à Berlin, les diplomates déplacent la frontière d'une dizaine de kilomètres vers le sud et Mangalia sera roumaine. La question émeut peu les opinions publiques : à Bucarest on est alors surtout offusqué par la cession de la Bessarabie du Sud à la Russie, tandis qu'à Sofia on pleure le rêve évanoui d'une Grande-Bulgarie. Quelques kilomètres de plus ou de moins de steppe dobroudjane ne constituent pas un enjeu. On note que l'Exarchat bulgare renonce immédiatement à la juridiction ecclésiastique sur la Dobroudja roumaine. D'autre part, les Nouveaux Territoires sont maintenus sous une juridiction spéciale et ne sont pleinement incorporés à la Roumanie qu'en 1913. La question des minorités linguistiques et religieuses se pose comme un défi aux États-nations. Au nord comme au sud, on procède à une véritable colonisation, afin de donner à ces territoires la « coloration nationale » requise.

Les choses s'enveniment véritablement en 1913, avec la Deuxième Guerre balkanique. L'intervention militaire de la Roumanie contre une Bulgarie dont la frontière septentrionale était complètement dégarnie scelle la cinglante défaite de cette 
dernière. Les négociations de paix ont lieu à Bucarest; la Roumanie y joue le rôle d'arbitre des conflits balkaniques et se réserve une compensation territoriale substantielle en s'octroyant la Dobroudja du Sud (le Quadrilatère), avec Tutrakan, Silistra, Dobrich et Balčik.

Les années 1913-1940 sont les pires dans les relations bulgaro-roumaines et la Dobroudja est au cœur de la polémique entre les deux pays. Le nationalisme roumain s'y montre sous son jour le plus déplaisant. Une fin est mise à cet épisode, finalement assez bref, par l'intervention du Troisième Reich, qui impose la restitution de la Dobroudja du Sud à la Bulgarie en septembre 1940. On procède alors à un échange de populations entre les deux pays. C'est un épisode assez peu connu de l'histoire balkanique, un des rares cas où cette mesure d'ingénierie ethnique radicale a véritablement résolu un contentieux politique. Le succès de cette opération tient sans doute à la population assez limitée contrainte à émigrer : 67000 Bulgares de Dobroudja du Nord et environ 80000 Roumains de Dobroudja du Sud (il faut rappeler ici l'importance de la composante turque dans la région, qui n'est pas concernée par l'échange de populations); d'autre part, cet échange a lieu dans un contexte de paix et on a pu mobiliser tous les moyens matériels et financiers pour le rendre le plus efficace; enfin, les migrants forcés ne sont pas déplacés sur une distance supérieure à 200 kilomètres et retrouvent donc dans leur nouveau lieu d'habitation, un climat et un environnement naturel qui leur sont familiers. Le thème des "patries perdues", si lancinant dans l'Europe d'après la Seconde Guerre mondiale, semble oublier la Dobroudja. Quand, après la chute du communisme, la plupart des contentieux balkaniques sont rouverts, on ne voit pas réémerger la question dobroudjane.

\section{Les fragments d'une histoire familiale}

Quittant les hauteurs de la géographie continentale et de l'histoire plurimillénaire, nous allons réduire la focale pour nous concentrer sur l'histoire d'une famille établie à Tulcea, dont nous pouvons suivre l'évolution entre 1850 et 1920 environ. Nous nous appuyons sur un document unique, ce qui est toujours hasardeux sur le plan méthodologique, et de plus, n'émanant pas d'une autorité scientifique très recommandable: Sultana Petrova. Elle fut en effet une dame de la cour de Ferdinand $\mathrm{I}^{\mathrm{er}}$ de Bulgarie, épouse du général Račo Petrov (ministre de la Guerre, ministre de l'Intérieur, Premier ministre de mai 1903 à octobre 1906 et un des principaux responsables de la désastreuse Seconde Guerre balkanique). Elle a laissé des mémoires qui sont une chronique mondaine, un recueil de potins assez divertissants. Elle a également rédigé quelques pages consacrées à l'histoire de sa famille, et en particulier à son grand-père maternel, publiées dans la revue Svobodna Dobrudža $\left(\mathrm{n}^{\circ} 40\right.$, 41,42 des 19 novembre, 10 et 17 décembre 1928). Derrière le snobisme et la superficialité de l'auteur et la roumanophobie de l'époque, on discerne certains mécanismes sociaux, révélateurs d'une époque où les stratégies de promotion sociale comptaient plus que des identités nationales encore mal définies.

Le roman familial commence peu après la guerre de Crimée avec l'installation à Tulcea des trois frères Teodorov. Ils sont originaires du gros village de Žeravna, près de Kotel, région connue pour ses commerçants entreprenants et influents, qui jouèrent un rôle important dans le mouvement national bulgare. Les trois frères disposent d'un certain capital pour s'installer. Le Bas-Danube est alors dans une phase de dynamisme 
économique, dont Brăila et GalaŢi sont les centres traditionnels, Sulina l'étoile montante et Tulcea le partenaire plus modeste, en territoire ottoman. Les trois frères bulgares se prénomment Dimitraki, Stefanaki et Perikli. Dimitraki Teodorov (né en 1822) épouse une Sultana (dont la sœur s'appelle Anika) et ils ont trois enfants qui sont baptisés Stefanaki, Telemah et Marionka. Cette dernière épouse Pantelej Minčovič (1834-1898) et ils auront trois enfants : Dimităr, Ljoljoka et Sultana, notre narratrice.

On est évidemment frappé par les prénoms très grecs portés dans cette famille supposée bulgare. La très forte hellénisation des élites urbaines dans la région du Bas-Danube est rarement évoquée dans les sources bulgares. Les origines bulgares, associées au monde rural, n'étaient alors pas valorisantes et l'ascension sur l'échelle sociale exigeait d'adopter la langue et les usages grecs.

Mais cela ne signifie pas pour autant une rupture irréversible. Les trois frères Teodorov restent attachés à leur village natal, où ils financent une fontaine en 1867, avec une longue inscription dédicatoire. Ils contribuent au financement du čitalište bulgare de Tulcea, ouvert en 1861. Ces salons de lecture, où l'on recevait les principaux journaux de l'époque, qui offraient une petite bibliothèque et où on pouvait se réunir pour discuter politique, organiser des causeries, etc. jouèrent un rôle très important dans la diffusion de l'idéologie nationale. L'évergétisme patriotique n'est pas une exclusivité de la cause grecque !

En revanche, il ne semble pas que la famille Teodorov ait noué de liens avec le monde aristocratique roumain. C'est qu'elle s'est installée sur la rive droite du Danube, en territoire ottoman.

Dimitraki Teodorov, connu comme Dimitraki-bey, entretient d'excellents rapports avec l'administration locale, le mutessarif chargé du sancak de Tulcea. Son frère, Stefanaki-bey, sera même député de la région à l'éphémère parlement ottoman de 1877. Le réseau familial a donc ses antennes dans la capitale et quand, en 1877, le gendre Pantelej Minčovič, qui est médecin militaire (un des rares secteurs de la fonction publique ottomane ouvert aux non-musulmans), risque de se faire muter à Alep, la famille est assez influente pour obtenir l'intervention $\mathrm{du}$ grand vizir Ahmed Vefik-Pasha.

30 Avec la guerre russo-turque de 1877-1878, les allégeances politiques sont mises à rude épreuve et il faut savoir louvoyer. Après une période d'incertitudes (on redoute des massacres et on agit pour que les autorités ottomanes assurent la protection des chrétiens), l'armée russe est accueillie avec enthousiasme par la famille Teodorov. Le gendre Minčovič accepte la fonction de préfet de Varna sous l'occupation russe.

31 Quelques mois plus tard, cette famille de notables provinciaux s'empresse avec le même zèle auprès des nouvelles autorités roumaines, incarnées par le général Matei Vlădescu. Le premier préfet, Georges Ghica, est un homme cultivé et courtois, avec lequel il est facile de s'entendre. Dimitraki-bey est nommé maire de Tulcea et il se retrouve au centre de la vie mondaine de la petite ville. Lors de sa première visite, le roi Carol se rend dans sa maison! Mais l'attitude envers la minorité bulgare se dégrade bientôt: sous le préfet Paul Statescu les titres de propriété sont mis en doute et des abus sont commis ; son successeur Ioan Neniţescu est un bulgarophobe déclaré.

32 La famille décide alors de se replier sur le territoire de la principauté de Bulgarie. Deux des frères, Stefanaki et Perikli, s'installent à Varna. Le gendre Minčovič fait sa carrière médicale à Sofia et se lance dans la politique: il sera même vice-président de 
l'Assemblée nationale en 1897-1898. Son fils, Dimităr Minčovič (1864-1944), le frère de Sultana, après des études au Robert College d'Istanbul, fait une carrière diplomatique : il est gérant de l'agence diplomatique bulgare de Bucarest en 1890-1891, puis en devient le titulaire officiel de 1895 à 1899 ; il occupera ensuite des postes à Rome et Londres.

Seul l'aîné, le patriarche Dimitraki Teodorov, s'obstine à rester en Dobroudja roumaine, et, dans l'impossibilité d'exercer un rôle social à la mesure de ses ambitions, il se renferme progressivement dans son domaine de Badila. Il exige la présence de ses deux petites-filles Ljolojka et Sultana à ses côtés. Tandis que leur frère fait ses études au Robert College, le meilleur établissement de tout l'Empire ottoman, les deux fillettes sont d'abord envoyées au pensionnat de Mme Papadopoulou à Galą̧i (dont leur mère avait été élève), puis, après avoir envisagé le Home School américain d'Istanbul, au pensionnat de Mme Borg, à Bucarest, une Allemande proche de la reine Élisabeth, où elles resteront six ans. Puis elles se marieront et mèneront la vie du high-life sofiote. Quoique plusieurs fois sollicité par ses frères et son gendre pour venir s'installer en Bulgarie, le vieux Dimitraki refusera toujours, et terminera ses jours pendant l'hiver 1885-1886, sur son domaine, comme une sorte de fossile social, vestige d'une période révolue dont il refuse de se séparer.

De ces fragments familiaux, on peut retenir qu'il y a eu un "moment grec» en Dobroudja du Nord, dans les années 1850-1870. Il est peut-être lié à l'activité de chantiers navals installés à Tulcea, qui exploitaient les forêts des monts de Măcin. Cette activité semble glisser vers GalaŢi et Brăila, mais surtout vers Sulina.

La famille est tentée de jouer la carte roumaine, mais ne s'insère pas vraiment dans les milieux de l'élite. Les petites-filles font certes leurs études dans un environnement gréco-roumain prestigieux. Mais une indication très claire signale que ce n'est pas la voie d'intégration souhaitée par la famille: leur tante Teodora, fille de Perikli Teodorov, ne doit à aucun prix épouser un Roumain!

C'est finalement l'option bulgare qui est retenue. Par sentiment patriotique, comme tente de nous convaincre Sultana Petrova dans ses souvenirs écrits dans l'entre-deux-guerres? Nous penchons plutôt pour des raisons plus pragmatiques. La principauté bulgare en voie d'édification offre des possibilités d'ascension sociale bien plus attrayantes : des postes à responsabilité pour des hommes ayant reçu une bonne éducation, même fort jeunes (p.ex. le frère Dimităr), des alliances matrimoniales prometteuses pour les filles, ce dont Sultana est la plus éclatante démonstration! Ces choix ne sont pas monolithiques: le vieux grand-père portera jusqu'à sa mort le fez ottoman, le petit-fils ambitieux saura négocier sa connaissance du roumain pour se construire une belle carrière diplomatique.

Quant à la Dobroudja, elle reste pour Sultana un berceau mythique, l'eldorado des années d'enfance, mais guère plus... 


\section{RÉSUMÉS}

La Dobroudja est une région où les relations roumano-grecques ont été particulièrement intenses. C'est, à l'échelle de l'Europe, une zone de contacts géographiques et historiques très spécifique.

Dans une optique macrogéographique, l'article esquisse plusieurs approches possibles: une Dobroudja danubienne, pontique, steppique ou carpatique. Selon les époques, les moyens de transport, les échanges commerciaux, les contraintes étatiques, ces différentes approches ont pesé sur le destin de la région.

La Dobroudja est ensuite considérée sous l'angle de la domination politique. Elle a longtemps été dominée par une politique de comptoirs maritimes, tant dans l'Antiquité qu'au Moyen-Âge. Dans une logique impériale, on constate que le Danube a assez systématiquement constitué une limite septentrionale d'expansion, ce qui met la Dobroudja dans une position de marginalité. Dans le conflit entre l'Empire russe et l'Empire ottoman elle se transforme au contraire en terrain d'affrontement préféré. La logique des États-nations à partir de la fin du XIX siècle place la Dobroudja au cœur du différend roumano-bulgare, et, pour le résoudre, on recourra à la mesure la plus extrême que les nationalismes aient mise au point: l'échange de populations. Il est à remarquer que l'échange de 1940 s'est révélé beaucoup moins traumatisant que d'autres.

Dans une troisième partie, on suit le destin d'une famille de notables provinciaux, les Teodorov. Originaires de Bulgarie orientale, ils s'installent à Tulcea après la guerre de Crimée et semblent avoir connu une forte hellénisation pendant une vingtaine d'années. Les revirements politiques en 1877-1878 remettent en question les allégeances nationales et une partie de la famille retourne vivre en Bulgarie, tandis que certains de ses membres restent en Roumanie.

Dobrudzha is a region where Romano-Greek contacts have been very active. As a whole, it is a place of geographical and historical contacts in Europe.

This paper considers different approaches in a geographical overview: Dobrudzha as part of a danubian, pontic, steppic or carpathic ensemble. At different times, when different ways of transportation are used for economic exchanges, and under different political constraints, the region played different roles.

A second part considers Dobrudzha according to political power. It has been controlled for a long time by thalassocraties, during the Antique and Medieval periods. When included into empires, Dobrudzha was left as a periphery, as the Danube was constantly a northern border. It became the favorite battleground for the Russian and the Ottoman empires in the XVIII-XIX ${ }^{\text {th }}$ centuries. With the emergence of the national states, Dobrudzha became an apple of discontent between Romania and Bulgaria. In order to solve it the most dramatic solution ever invented by nationalism was applied: an exchange of population, which, strangely enough, did not cause any noticeable embitterment.

The third part is devoted to a family of prominent provincialists, the Teodorovs. They came from Eastern Bulgaria to Tulcea after the Crimean war and seem to have undergone a quick Hellenization during twenty years. With the political turning point of 1877-1878 their national choices change: part of the family settles in Bulgaria, when the other stays in Romania.

Dobrogea este o regiune în care relaŢiile greco-române au fost foarte intense. La scara Europei, este o zonă de contacte geografice şi istorice cu totul deosebită. Din punct de vedere macro-geografic, studiul schiŢează mai multe perspective posibile: o Dobroge danubiană, pontică, de stepă sau carpatică. În funcţie de epoci, de mijloacele de transport, de schimburile comerciale, de constrângerile statale, aceste perspective au influenţat destinul regiunii. 
Dobrogea este apoi privită sub unghiul dominaţiei politice. A fost dominată multă vreme de aç̧iunea stabilimentelor de comerȚ maritim, atât în perioada antică cât şi în Evul Mediu. Într-o logică imperială, vedem că Dunărea a fost în mod destul de sistematic o limită nordică pentru expansiune, ceea ce plasează Dobrogea într-o situaŢie de marginalitate. În schimb, în înfruntarea între Imperiul rus şi Imperiul otoman, aceasta se transformă în teren de luptă preferat. Începând cu sfârşitul secolului al xIX-lea, logica statelor-naŢiuni situează Dobrogea în centrul conflictului româno-bulgar iar pentru a-l rezolva se va recurge la soluţia cea mai extremă imaginată de naŢionalişti : schimbul de populaţie. De remarcat este faptul că schimbul din 1940 a fost mult mai puŢin traumatizant decât altele.

În partea a treia urmărim destinul unei familii de notabili provinciali, familia Teodorov. Originari din Bulgaria orientală, aceştia se stabilesc la Tulcea după războiul Crimeii şi par să fi cunoscut o elenizare puternică timp de douăzeci de ani. Răsturnările politice din 1877-1878 pun sub semnul întrebării subordonările naţionale şi o parte din familie se întoarce în Bulgaria în timp ce unii dintre membrii ei rămân în România.

\section{INDEX}

motsclesmk ГРЦИЈА/РОМАНИЈА ОДНОСИ, РОМАНИЈА, ДОБРУЏА, ДЕВЕТНАЕСЕТТИОТ ВЕК, ДВАЕСЕТТИОТ ВЕК, ИСТОРИЈА, ГЕОПОЛИТИКА, МЕМОАРИТЕ

motsclestr Yunan-Romen ilişkileri, Romanya, Dobruca, Ondokuzuncu yüzyıl, Yirminci yüzyıl, Tarih, Jeopolitik, Anıları

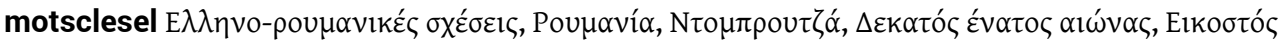

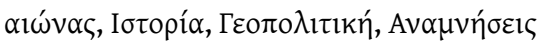

Mots-clés : relations gréco-roumaines, relations gréco-roumaines

Index géographique : Roumanie, Dobroudja

Thèmes : Histoire, géopolitique, mémoires

Keywords : Greek-Romanian relations, Romania, Dobruja, Nineteenth century, Twentieth century, History, Geopolitics, Memories

Index chronologique : dix-neuvième siècle, vingtième siècle

\section{AUTEUR}

\section{BERNARD LORY}

CREE/Inalco/USPC 